\title{
Brentuximab vedotin for relapsed or refractory CD30+ Hodgkin lymphoma: a multicenter analysis from Asia
}

This article was published in the following Dove Press journal:

OncoTargets and Therapy

26 September 2014

Number of times this article has been viewed

\section{Qing-Ming Yang 1 ,* \\ Jung Yong Hong 2,* \\ Young Hyeh $\mathrm{Ko}^{3}$ \\ Shek-Ying $\operatorname{Lin}^{4}$ \\ Wing-Yan $\mathrm{Au}^{4}$ \\ Moon Ki Choi \\ Silvia Park ${ }^{6}$ \\ Seok Jin $\mathrm{Kim}^{6}$ \\ Won Seog Kim ${ }^{6}$}

'The First Affiliated Hospital of the People's Liberation Army General Hospital, Beijing, People's Republic of China; ${ }^{2}$ Department of Internal Medicine, Chung-Ang University College of Medicine, ${ }^{3}$ Department of Pathology, Samsung Medical Center, Sungkyunkwan University School of Medicine, Seoul, Korea; ${ }^{4}$ Blood-Med Clinic, Hong Kong, People's Republic of China; ${ }^{5}$ Divisions of Hematology and Medical Oncology, Department of Internal Medicine, Seoul National University Bundang Hospital, Seoul National University College of Medicine, Gyeonggi-do, Korea; ${ }^{6}$ Division of Hematology-Oncology, Department of Medicine, Samsung Medical Center, Sungkyunkwan University School of Medicine, Seoul, Korea

*Qing-Ming Yang and Jung Yong Hong contributed equally to this work

Correspondence: Won Seog Kim Division of Hematology-Oncology, Department of Medicine, Samsung Medical Center, Sungkyunkwan University School of Medicine, 50 Irwon-dong Gangnam-gu, Seoul, I35-710, Korea

$\mathrm{Tel}+82234106548$

Fax +82 234101754

Email wskimsmc@skku.edu
Introduction: Brentuximab vedotin (SGN-35), an anti-cluster of differentiation (CD)-30 antibody conjugated to the anti-tubulin agent monomethyl auristatin $\mathrm{E}$, has demonstrated promising efficacy and tolerability in relapsed and heavily treated Hodgkin lymphoma (HL). In this study, we report the Asian experience with brentuximab vedotin in patients with relapsed or refractory CD30-positive (CD30+) HL.

Methods: This is an observational, multicenter, retrospective study. Between October 2011 and June 2013, a total of 22 patients were treated with brentuximab vedotin under a named patient program in Asia. Patients received a 30 min infusion of brentuximab vedotin at a dose of 1.8 $\mathrm{mg} / \mathrm{kg}$ of body weight every 3 weeks.

Results: Four patients (18.2\%) showed a complete response, and the overall response rate was $72.7 \%$. The median duration of response was 4.4 months (range 1.0-17.4). The median progression-free survival was 5.7 months, and the median overall survival has not yet been reached. The 1-year expected survival rate was $67.2 \%$. The most common grade $3 / 4$ adverse events were neutropenia ( $n=7 ; 31.8 \%$ ). No patients experienced grade $3 / 4$ sensory neuropathy.

Conclusions: These results confirm that brentuximab vedotin as a single agent is also effective and well tolerated when used in Asian patients with relapsed and refractory CD30+ HL.

Keywords: Asian, efficacy, safety

\section{Background}

Therapeutic advances in the treatment of Hodgkin lymphoma (HL) have been achieved with improvements in combined approaches such as chemotherapy and radiotherapy. The long-term survival rate of $\mathrm{HL}$ is now $70 \%-90 \% .{ }^{1,2}$ However, depending on the disease status, $5 \%-30 \%$ of patients with HL relapse or have refractory disease after frontline treatment. The standard treatment for these relapsed or refractory patients is salvage chemotherapy followed by autologous hematopoietic stem cell transplantation (HSCT), which can induce durable remission in up to $50 \%$ of these patients. ${ }^{3}$ However, patients who experience relapse or progression after autologous HSCT have a grave prognosis, with a median overall survival (OS) of 1-2 years. ${ }^{4}$

Classical HL is characterized by the presence of a cluster of differentiation-30positive (CD30+) Reed-Sternberg or Hodgkin cells in a matrix of inflammatory cells. Brentuximab vedotin (SGN-35), an anti-CD30 antibody conjugated to the anti-tubulin agent monomethyl auristatin E, has demonstrated promising anti-tumor activity in relapsed and heavily treated HL patients, with an overall objective response rate of $60 \%-75 \% .^{5-8}$ Based on these impressive clinical data, the US Food and Drug Administration recently approved brentuximab vedotin for classical HL patients who 
have relapsed disease after autologous HSCT or who have progressive disease after at least two lines of multi-agent chemotherapy and are not suitable candidates for autologous HSCT.

HL shows unique ethnic and regional distributions; that is, it is diagnosed less frequently in Asian than in Western countries. ${ }^{9-12}$ Most data on brentuximab vedotin in relapsed or refractory classical HL patients have been reported from Western countries, and data on the clinical efficacy of brentuximab vedotin in Asian patients are scarce as present. Here, we report an Asian experience with brentuximab vedotin in patients with relapsed and/or refractory CD30+ classical HL.

\section{Methods}

\section{Trial design and patients}

An observational, multicenter, retrospective analysis was performed to investigate the efficacy and toxicity of brentuximab vedotin in Asian patients with relapsed and/or refractory CD30+ classical HL. A total of 22 patients were treated with brentuximab vedotin between October 2011 and June 2013, under a named-patient program (NPP) in Asia. All patients were pathologically confirmed to be CD30+ in immunohistochemistry analysis. The patients with nodular lymphocyte-predominant HL and CD30+ anaplastic largecell lymphoma (ALCL) were excluded from this study.

\section{Treatment response and toxicity assessment}

Patients received a $30 \mathrm{~min}$ infusion of brentuximab vedotin at a dose of $1.8 \mathrm{mg} / \mathrm{kg}$ of body weight every 3 weeks. Treatment was continued until disease progression, unacceptable toxicity, or the patient refused to continue. Treatment responses were evaluated every one or two cycles of chemotherapy by computed tomography and/or positron-emission tomography (PET) scan using the revised response criteria for malignant lymphoma. ${ }^{13}$ All adverse events were evaluated according to the National Cancer Institute Common Terminology Criteria for Adverse Events (NCI-CTCAE) version 4.0. Dose modification of brentuximab vedotin was based on toxicities on the day of treatment according to the system showing the greatest toxicities.

\section{Statistical analysis}

Descriptive statistics are reported as proportions and medians. Duration of response is calculated from the day of response achievement to disease progression. Progression-free survival (PFS) was calculated from initiation of brentuximab vedotin to the first day of disease progression, relapse, or death from any cause. OS was calculated from initiation of brentuximab vedotin to death. PFS and OS were censored on the last date of follow-up. Survival curves were estimated using the Kaplan-Meier method. Statistical analyses were performed using the software package IBM PASW (version 18.0; SPSS Inc., Chicago, IL, USA).

\section{Results}

\section{Patient characteristics}

Table 1 shows the baseline clinical characteristics of the 22 patients at the time of diagnosis of classical HL. The median age was 30 years (range 16-57), and the male-tofemale ratio was 15:7. A total of 12 patients $(54.5 \%)$ had classical HL, with nodular sclerosis as the most frequent subtype; eight (36.4\%) had mixed-cellularity HL. Of the 22 patients, $12(54.5 \%)$ showed primary refractory disease and ten (45.5\%) showed relapsed disease status. Most of the patients had received three or more lines of previous chemotherapy $(n=20 ; 90.9 \%)$ and/or HSCT $(n=17 ; 77.3 \%)$.

\section{Efficacy}

The median number of brentuximab vedotin cycles was five (range 1-18). The response and survival outcomes are summarized in Table 2. Of the 22 patients in the study, one patient $(4.5 \%)$ was not evaluable for a treatment response. As an intention-to-treat analysis, four patients (18.2\%) showed a complete response (CR) and 12 (54.5\%) showed a partial response (PR), resulting in an overall response (OR) rate of $72.7 \%$. The median duration of response was 4.4 months (range 1.0-17.4), and the median time to response was 0.9 months (range $0.7-3.0$ ). Of the 22 patients, 16 were alive at the time of data collection. The median PFS was 5.7 months, and the median OS has not yet been reached. The 1-year expected survival rate was 67.2\% (Figure 1). The PFS according to the achieved response showed a statistically significant difference, and the survival curves are demonstrated in Figure 2.

\section{Safety}

Treatment-related adverse events are summarized in Table 2. One patient died of pneumonia after one cycle of brentuximab vedotin. The most common grade $3 / 4$ adverse event was neutropenia ( $n=7 ; 31.8 \%$ ). Grade $3 / 4$ non-hematological adverse events observed were pneumonia $(n=1)$, hemorrhagic cystitis $(n=1)$, nausea $(n=1)$, constipation $(n=1)$, and muscle pain $(n=1)$. No patient developed grade $3 / 4$ sensory neuropathy or progressive multifocal leukoencephalopathy. 
Table I Baseline characteristics and previous treatment history $(n=22)$

\begin{tabular}{|c|c|}
\hline Variables & $\begin{array}{l}n(\%) \text { or } \\
\text { median (range) }\end{array}$ \\
\hline Age, years & $30(16-57)$ \\
\hline \multicolumn{2}{|l|}{ Sex } \\
\hline Male & $15(68.2)$ \\
\hline Female & $7(31.8)$ \\
\hline \multicolumn{2}{|l|}{ ECOG performance } \\
\hline PS 0-I & $10(45.5)$ \\
\hline PS $2-4$ & $12(54.5)$ \\
\hline \multicolumn{2}{|l|}{ B-symptoms } \\
\hline No & $7(31.8)$ \\
\hline Yes & I5 (68.2) \\
\hline \multicolumn{2}{|l|}{ Extranodal involvement } \\
\hline $0-1$ & $14(63.6)$ \\
\hline 2 or more & $8(36.4)$ \\
\hline \multicolumn{2}{|l|}{ LDH elevated } \\
\hline No & I5 (68.3) \\
\hline Yes & $7(31.8)$ \\
\hline \multicolumn{2}{|l|}{ Stage } \\
\hline I-II & $\mathrm{I}(4.5)$ \\
\hline III-IV & $21(95.5)$ \\
\hline \multicolumn{2}{|l|}{ Pathology } \\
\hline NSHL & $12(54.5)$ \\
\hline $\mathrm{MCHL}$ & $8(36.4)$ \\
\hline LRHL & I (4.5) \\
\hline $\mathrm{CHL}$, unspecified & I (4.5) \\
\hline \multicolumn{2}{|l|}{ Nationality } \\
\hline China & $14(63.6)$ \\
\hline South Korea & $6(27.3)$ \\
\hline Hong Kong & $2(9.1)$ \\
\hline \multicolumn{2}{|l|}{ Disease status } \\
\hline Primary refractory disease & $12(54.5)$ \\
\hline Relapsed disease & $10(45.5)$ \\
\hline \multicolumn{2}{|l|}{ Previous chemotherapy lines } \\
\hline $\mathrm{I}-2$ & $2(9.1)$ \\
\hline 3 or more & $20(90.9)$ \\
\hline \multicolumn{2}{|l|}{ Previous HSCT } \\
\hline No & $5(22.7)$ \\
\hline Yes (auto and/or allo) & $17(77.3)$ \\
\hline Years between initial Dx and brentuximab & $3.4(1.2-16.6)$ \\
\hline Cycles of brentuximab & $5(I-18)$ \\
\hline
\end{tabular}

Abbreviations: CHL, classical Hodgkin lymphoma; Dx, diagnosis; ECOG, Eastern Cooperative Oncology Group; HSCT, hematopoietic stem cell transplantation; LDH, lactic dehydrogenase; LRHL, lymphocyte-rich Hodgkin lymphoma; MCHL, mixedcellularity Hodgkin lymphoma; NSHL, nodular sclerosis Hodgkin lymphoma; PS, performance status.

\section{Discussion}

German, British, and Italian groups recently published consistent clinical data showing the high efficacy and manageable toxicity of brentuximab vedotin in patients with relapsed and refractory $\mathrm{CD} 30+\mathrm{HL}$, which support the previous global phase II study. ${ }^{6}{ }^{614-16}$ However, of the population in the pivotal phase II study by Younes et al, ${ }^{6}$ only $7 \%$ were Asian patients, and independent Asian data on the efficacy and safety of brentuximab vedotin have not yet been validated. Only one
Table 2 Response results and toxicity profiles

\begin{tabular}{|c|c|c|}
\hline Measure & $\begin{array}{l}\text { No of } \\
\text { patients (\%) }\end{array}$ & $95 \% \mathrm{Cl}$ \\
\hline \multicolumn{3}{|l|}{ Best response } \\
\hline Complete response & $4(18.2)$ & $2.1-34.3$ \\
\hline Partial response & $12(54.5)$ & $33.7-75.3$ \\
\hline Stable disease & $4(18.2)$ & $2.1-34.3$ \\
\hline Progressive disease & I (4.5) & $0.0-13.6$ \\
\hline Not available & I (4.5) & $0.0-13.6$ \\
\hline Objective response & $16(72.7)$ & $54.1-91.3$ \\
\hline $\begin{array}{l}\text { Duration of objective response, } \\
\text { months (range) }\end{array}$ & $4.4(1.0-17.4)$ & NA \\
\hline \multicolumn{3}{|l|}{ Survival outcome } \\
\hline Median PFS, months & 5.7 & NA \\
\hline Median OS, months & NR & NA \\
\hline I-year expected survival rate, \% & 67.2 & NA \\
\hline \multicolumn{3}{|l|}{ Toxicity profiles ${ }^{\mathrm{b}}$ (grade $3 / 4$ ) } \\
\hline \multicolumn{3}{|l|}{ Hematologic } \\
\hline $\begin{array}{l}\text { Neutropenia (neutrophil } \\
<1,000 / \mathrm{mm}^{3} \text { ) }\end{array}$ & $7(31.8)$ & $|2.3-5| .3$ \\
\hline $\begin{array}{l}\text { Thrombocytopenia (platelet } \\
<50,000 / \mathrm{mm}^{3} \text { ) }\end{array}$ & $3(13.6)$ & $0.0-27.9$ \\
\hline \multicolumn{3}{|l|}{ Non-hematologic } \\
\hline Pneumonia & $\mathrm{I}(4.5)$ & $0.0-13.6$ \\
\hline Hemorrhagic cystitis & $\mathrm{I}(4.5)$ & $0.0-13.6$ \\
\hline Nausea & I (4.5) & $0.0-13.6$ \\
\hline Constipation & I (4.5) & $0.0-13.6$ \\
\hline Muscle pain & I (4.5) & $0.0-13.6$ \\
\hline Sensory neuropathy & $0(0)$ & NA \\
\hline Dose reduction of brentuximab & $4(\mid 8.2)$ & $2.1-34.3$ \\
\hline
\end{tabular}

Notes: annless otherwise indicated; btoxicity profiles were evaluated according to National Cancer Institute Common Terminology Criteria for Adverse Events (NCl-CTCAE) 4.0.

Abbreviations: $\mathrm{Cl}$, confidence interval; NA, not applicable; NR, not reached; $\mathrm{OS}$, overall survival; PFS, progression free survival.

Japanese series recently showed that brentuximab vedotin had an acceptable safety profile and promising anti-tumor activity in 14 patients with relapsed or refractory CD30+ HL and ALCL. ${ }^{17}$ Considering the differences in ethnic and regional distribution of HL between Asian and Western countries, we performed this study to provide information about the efficacy and safety of brentuximab vedotin in Asian HL patients.

The risk profile of patients in our study was similar to that of patients in the previous series. ${ }^{6,8,14-16}$ Baseline characteristics, such as median age (30 years), primary refractory disease $(54.5 \%)$, and prior SCT $(77.3 \%)$, were also similar. Our series showed a male predominance (68.2\%) and a high percentage of patients with advanced stage at diagnosis $(95.5 \%$ stage III-IV). In terms of efficacy, our results showed an OR rate of $72.7 \%$ and a CR rate of $18.2 \%$. These findings confirmed the high efficacy of brentuximab vedotin reported in the previous series, which reported an OR rate of $60 \%-73 \%$ and a CR rate of $17 \%-32 \%{ }^{6,14-17}$ 

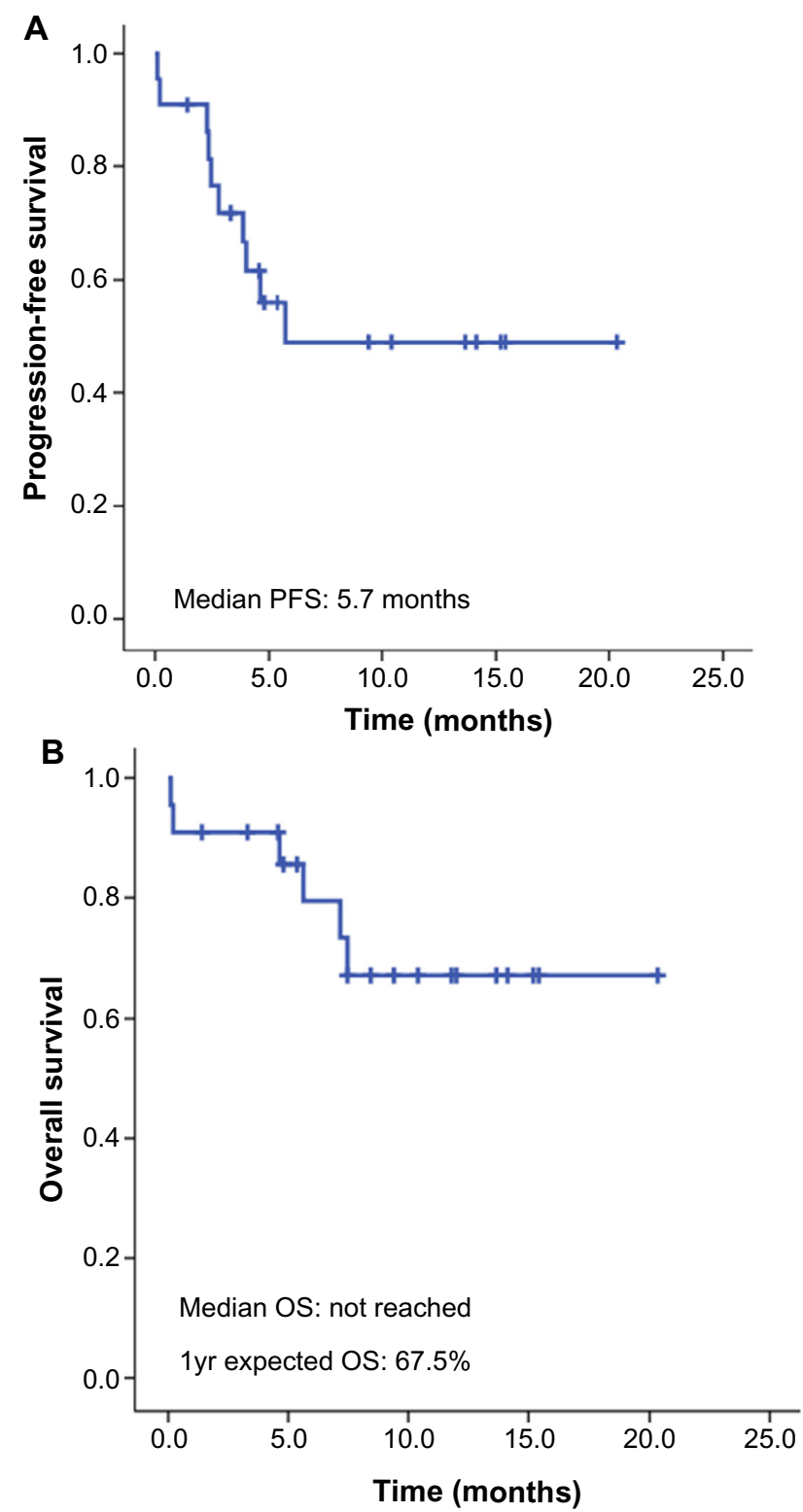

Figure I Survival of patients.

Notes: (A) Progression-free survival; (B) overall survival.

Abbreviations: OS, overall survival; PFS, progression-free survival.

With regard to toxicity profile, most adverse events could be managed through standard supportive care. The most common grade $3 / 4$ adverse event was neutropenia ( $n=7$; $31.8 \%$ ), which was a higher frequency than that reported in the Western series. ${ }^{14-16}$ However, the incidence of grade 3/4 neutropenia in a recent Japanese series (15\%) was comparable to that in the Western series. ${ }^{17}$ Therefore, whether Asian populations are vulnerable to neutropenia with brentuximab vedotin is still inconclusive, and further studies are needed. The previous pivotal phase II trial reported that peripheral neuropathy was a common adverse event associated with brentuximab vedotin. ${ }^{6}$ The German and the Japanese groups
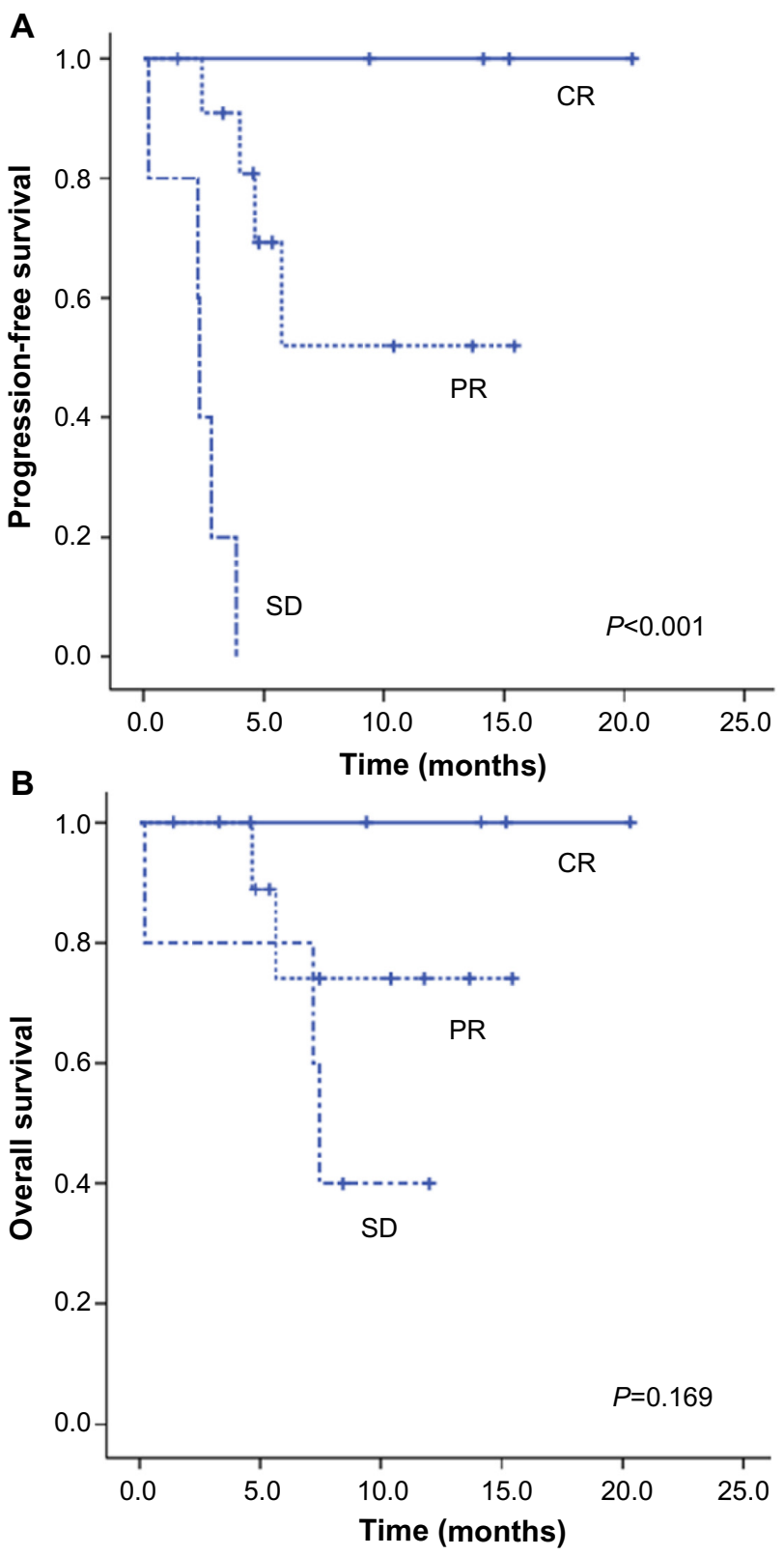

Figure 2 Survival of patients according to response to brentuximab vedotin. Notes: (A) Progression-free survival; (B) overall survival.

Abbreviations: $C R$, complete response; PR, partial response; SD, stable disease.

observed no patients with grade $3 / 4$ peripheral neuropathy. ${ }^{14,17}$ However, grade $1 / 2$ peripheral neuropathy was common $(41 \%-60 \%) .^{14,17}$ This study also observed that no patients had grade $3 / 4$ peripheral neuropathy. However, grade 1/2 peripheral neuropathy was not fully evaluated due to the retrospective nature of this study. A recent phase I trial of brentuximab vedotin plus ABVD (adriamycin, bleomycin, vinblastine, and dacarbazine) by Younes et a $1^{18}$ reported significant pulmonary toxic events (36\%). Of note, one case in this study died of acute respiratory distress syndrome and pneumonia after the first cycle of brentuximab vedotin. In the 
Japanese series, two patients experienced serious pulmonary adverse events, including pneumocystis jirovecii pneumonia and pneumonia with unknown etiology. ${ }^{17}$ Therefore, further studies are needed to investigate whether frequent significant pulmonary toxic effects are caused by brentuximab vedotin alone or only in combination with bleomycin.

Several clinical trials regarding the efficacy of brentuximab vedotin are ongoing in diverse clinical settings. Chen et $\mathrm{al}^{19}$ showed that brentuximab vedotin may provide sufficient disease control to enable reduced-intensity allogeneic HSCT, and brentuximab vedotin before allogeneic HSCT did not appear to adversely affect engraftment, graft-versus-host disease, or survival. Sasse et $\mathrm{al}^{20}$ reported on the therapeutic efficacy of brentuximab vedotin in chemotherapy-refractory and transplant-naïve HL patients. Now, a randomized, double-blind, phase III trial is ongoing comparing brentuximab vedotin plus AVD (Adriamycin, vinblastine, and dacarbazine) versus ABVD for the first-line treatment of advanced classical HL (ECHELON-1) (clinicaltrials.gov identifier: NCT01712490). A randomized, double-blind, phase III trial is also comparing the efficacy and safety of brentuximab vedotin (SGN-35) plus best supportive care compared with placebo plus best supportive care in treatment of residual HL following autologous HSCT (AETHERA) (clinicaltrials.gov identifier: NCT01100502); the results of these studies are anticipated.

The present study represents the largest independent set of Asian data for HL patients treated with brentuximab vedotin in a non-trial setting. The results confirm the previously reported high efficacy and acceptable toxicity of brentuximab vedotin in patients with relapsed and refractory CD30+ classical HL. Although these clinical data for brentuximab vedotin in HL are impressive, further clinical trials are needed to assess long-term survival and unexpected complications.

\section{Acknowledgment}

The authors thank the staff of all centers that participated in data collection.

\section{Authors' contributions}

All authors contributed toward data analysis, drafting and revising the paper and agree to be accountable for all aspects of the work.

\section{Disclosure}

The authors have no conflicts of interest in this work.

\section{References}

1. Kuruvilla J. Standard therapy of advanced Hodgkin lymphoma. Hematology Am Soc Hematol Educ Program. 2009:497-506.

2. Connors JM. State-of-the-art therapeutics: Hodgkin's lymphoma. J Clin Oncol. 2005;23(26):6400-6408.

3. Majhail NS, Weisdorf DJ, Defor TE, et al. Long-term results of autologous stem cell transplantation for primary refractory or relapsed Hodgkin's lymphoma. Biol Blood Marrow Transplant. 2006;12(10): $1065-1072$.

4. von Tresckow B, Engert A. The role of autologous transplantation in Hodgkin lymphoma. Curr Hematol Malig Rep. 2011;6(3): $172-179$.

5. Fanale MA, Forero-Torres A, Rosenblatt JD, et al. A phase I weekly dosing study of brentuximab vedotin in patients with relapsed/ refractory CD30-positive hematologic malignancies. Clin Cancer Res. 2012;18(1):248-255.

6. Younes A, Gopal AK, Smith SE, et al. Results of a pivotal phase II study of brentuximab vedotin for patients with relapsed or refractory Hodgkin's lymphoma. J Clin Oncol. 2012;30(18):2183-2189.

7. Younes A, Bartlett NL, Leonard JP, et al. Brentuximab vedotin (SGN-35) for relapsed CD30-positive lymphomas. N Engl J Med. 2010;363(19):1812-1821.

8. Monjanel H, Deville L, Ram-Wolff C, et al. Brentuximab vedotin in heavily treated Hodgkin and anaplastic large-cell lymphoma, a single centre study on 45 patients. Br J Haematol. 2014;166(2):306-308.

9. Shin HR, Masuyer E, Ferlay J, Curado MP; Asian Contributors to CI5 IX4. Cancer in Asia - Incidence rates based on data in cancer incidence in five continents IX (1998-2002). Asian Pac J Cancer Prev. 2010; 11 Suppl 2:11-16.

10. Won YW, Kwon JH, Lee SI, et al. Clinical features and outcomes of Hodgkin's lymphoma in Korea: Consortium for Improving Survival of Lymphoma (CISL). Ann Hematol. 2012;91(2):223-233.

11. Park HJ, Park EH, Jung KW, et al. Statistics of hematologic malignancies in Korea: incidence, prevalence and survival rates from 1999 to 2008. Korean J Hematol. 2012;47(1):28-38.

12. Huh J. Epidemiologic overview of malignant lymphoma. Korean $J$ Hematol. 2012;47(2):92-104.

13. Cheson BD, Pfistner B, Juweid ME, et al. Revised response criteria for malignant lymphoma. J Clin Oncol. 2007;25(5):579-586.

14. Rothe A, Sasse S, Goergen H, et al. Brentuximab vedotin for relapsed or refractory $\mathrm{CD} 30+$ hematologic malignancies: the German Hodgkin Study Group experience. Blood. 2012;120(7):1470-1472.

15. Gibb A, Jones C, Bloor A, et al. Brentuximab vedotin in refractory CD30+ lymphomas: a bridge to allogeneic transplantation in approximately one quarter of patients treated on a Named Patient Programme at a single UK center. Haematologica. 2013;98(4):611-614.

16. Zinzani PL, Viviani S, Anastasia A, et al. Brentuximab vedotin in relapsed/refractory Hodgkin's lymphoma: the Italian experience and results of its use in daily clinical practice outside clinical trials. Haematologica. 2013;98(8):1232-1236.

17. Ogura M, Tobinai K, Hatake K, et al. Phase I/II study of brentuximab vedotin in Japanese patients with relapsed or refractory CD30-positive Hodgkin's lymphoma or systemic anaplastic large-cell lymphoma. Cancer Sci. 2014;105(7):840-846.

18. Younes A, Connors JM, Park SI, et al. Brentuximab vedotin combined with ABVD or AVD for patients with newly diagnosed Hodgkin's lymphoma: a phase 1, open-label, dose-escalation study. Lancet Oncol. 2013;14(13):1348-1356.

19. Chen R, Palmer JM, Thomas SH, et al. Brentuximab vedotin enables successful reduced-intensity allogeneic hematopoietic cell transplantation in patients with relapsed or refractory Hodgkin lymphoma. Blood. 2012;119(26):6379-6381.

20. Sasse S, Rothe A, Goergen H, et al. Brentuximab vedotin (SGN-35) in patients with transplant-naive relapsed/refractory Hodgkin lymphoma. Leuk Lymphoma. 2013;54(10):2144-2148. 


\section{Publish your work in this journal}

OncoTargets and Therapy is an international, peer-reviewed, open access journal focusing on the pathological basis of all cancers, potential targets for therapy and treatment protocols employed to improve the management of cancer patients. The journal also focuses on the impact of management programs and new therapeutic agents and protocols on

patient perspectives such as quality of life, adherence and satisfaction. The manuscript management system is completely online and includes a very quick and fair peer-review system, which is all easy to use. Visit http://www.dovepress.com/testimonials.php to read real quotes from published authors.

Submit your manuscript here: http://www.dovepress.com/oncotargets-and-therapy-journal 